\title{
Los hábitos de estudio y el rendimiento académico de los estudiantes del nivel secundario
}

\section{Study habits and academic performance of secondary school students}

\author{
Susan Manrique; Edwin Estrada ${ }^{2}$
}

\section{RESUMEN}

La presente investigación tuvo como objetivo determinar la relación que existe entre los hábitos de estudio y el rendimiento académico de los estudiantes del nivel secundario de la Institución Educativa 52191 "Santo Domingo de Guzmán" del distrito de Laberinto, Tambopata. El enfoque de investigación fue cuantitativo, el tipo de investigación fue no experimental y el diseño fue descriptivo, correlación y transeccional.

La población de estudio fue conformada por todos los estudiantes del nivel secundario de la Institución Educativa 52191 "Santo Domingo" y la muestra por 116 estudiantes, cantidad que fue obtenida mediante un muestreo probabilístico estratificado. Para la recolección de datos se utilizó el Inventario de Hábitos de Estudio CASM-85. Revisión-98 y el acta consolidada de evaluación integral del nivel secundario. Los resultados muestran que estadísticamente existe correlación alta, positiva y significativa entre las variables de estudio. El coeficiente de correlación $r$ de Pearson es de 0,843 con un $p$-valor inferior al nivel de significancia $(p=0,000<0,05)$. Finalmente se concluye que mientras los estudiantes tengan mejores hábitos de estudio, su rendimiento académico será el más óptimo.

Palabras clave: Hábitos de estudio; rendimiento académico; estudio.

\section{ABSTRACT}

The purpose of this research was to determine the relationship between the study habits and the academic performance of the students of the secondary level of the 52191 Educational Institution "Santo Domingo de Guzmán" of the district of Laberinto, Tambopata. The research approach was quantitative, the type of research was non-experimental and the design was descriptive, correlation and transectional.

The study population was made up of all the students of the secondary level of the Educational Institution 52191 "Santo Domingo" and the sample by 116 students, an amount that was obtained through a stratified probabilistic sampling. The CASM-85 Study Habits Inventory was used to collect data. Revision-98 and the consolidated record of comprehensive evaluation of the secondary level. The results show that statistically there is a high, positive and significant correlation between the study variables. Pearson's correlation coefficient $r$ is 0.843 with a p-value below the level of significance $(p=0.000<0.05)$. Finally, it is concluded that as long as the students have better study habits, their academic performance will be the most optimal.

Keywords: Study habits; academic performance; study. 


\section{INTRODUCCIÓN}

En la actualidad todo estudiante tiene el reto de desarrollar sus competencias, habilidades y destrezas académicas a lo largo de toda su vida escolar. Para ello el docente realiza acciones que buscan desarrollar integralmente a los estudiantes, sin embargo, es necesario también fomentar que ellos actúen autónoma y reflexivamente.

En el caso de los estudiantes que se encuentran cursando el nivel secundario, es necesario que alcancen un nivel de madurez que les posibilite ser reflexivo sobre cómo desarrollar sus propios aprendizajes, qué estrategia utilizar para obtener mejores resultados y qué necesitan aprender prioritariamente y no quedarse desfasados.

No obstante, la experiencia en la práctica pedagógica desarrollada en la institución educativa "Santo Domingo de Guzmán" permite ver que los estudiantes desconocen qué acciones deben realizar autónomamente para conseguir aprender, creen que el estudio es un procedimiento pasivo y que dependeremos siempre de otras personas para que aprendamos, es decir, desconocen las maneras pertinentes de cómo estudiar porque normalmente ellos están acostumbrados a que los docentes les dicten, formulen interrogantes estrictamente para memorizarlas sin hacer ningún esfuerzo que demande una disonancia cognitiva ni reflexionar sobre la importancia de lo que aprenden. Muchos de ellos se han acostumbrado a escuchar de forma pasiva y no intervenir en clase.

Lo manifestado previamente se agudiza al ver los resultados de los aprendizajes de los estudiantes. Prueba de ello son las pruebas censales, las cuales muestran niveles de logro de aprendizajes poco favorables. El año 2018 se aplicó la Evaluación Censal de Estudiantes (ECE) a estudiantes de segundo grado del nivel secundario y los resultados evidencian que, en promedio, la tercera parte de estudiantes se encuentra en el nivel previo al inicio, en lo que respecta a Matemática, Ciencia y Tecnología, Lectura y Ciencias Sociales. Por ello se asume que dicha problemática se puede generalizar a todo el nivel secundario puesto que en el año 2016 aproximadamente el 10\% ha alcanzado el nivel satisfactorio en las áreas de Historia, Geografía y Economía, Lectura y Matemática.

Por lo expuesto, este estudio se justifica ya que, a partir de los resultados que se obtendrán, los docentes y personal directivo de la institución podrán aplicar estrategias como talleres, charlas y se podrá trabajar desde el área de tutoría para el cumplimiento de la competencia transversal "Gestiona su aprendizaje de manera autónoma". En ese sentido se podrá proponer variadas técnicas y estrategias que se ajusten a las necesidades e intereses de los estudiantes para la puesta en práctica de diferentes hábitos de estudio. De esta manera mostrarán un mejor desempeño en el aula, lo que conllevará a un adecuado rendimiento académico.

De acuerdo a la revisión de la literatura, diversos autores han hallado en sus investigaciones que existe asociación entre los hábitos de estudio y el rendimiento académico (Chilca, 2017; Pineda y Alcántara, 2017; Grados y Alfaro, 2013; Espinoza, 2017; Escurra, Romero, Moreno, Ahumada, Juárez y Ramos, 2015; Yamada, Bazán y Espinoza, 2017).

A partir de la información expuesta, Cartagena (citado por Hernández, Rodríguez y Vargas, 2012) define a los hábitos de estudio como los métodos y estrategias que suelen utilizar los estudiantes para asimilar unidades de aprendizaje, su aptitud para eludir distractores, su atención al material específico y los esfuerzos que realiza durante todo el proceso.

Poves (citado por Cruz, 2011) menciona que un hábito de estudio es una acción que se realiza constantemente a las mismas horas. La reincidencia de este comportamiento durante el tiempo genera un mecanismo inconsciente.

Belaunde (1994) define que el concepto de hábito de estudio se refiere a la manera cómo el estu- 
diante afronta usualmente a su quehacer educativo, es la costumbre natural de buscar aprender constantemente, lo cual implica la manera en el estudiante se organiza en cuanto a tiempo, espacio, técnica y métodos, concretos que utiliza para estudiar.

Como en cualquier otra actividad, la habilidad y la dedicación son factores importantes para lograr aprendizajes. Los hábitos de estudio son los métodos y estrategias que acostumbra a usar el estudiante para asimilar conocimientos, su aptitud para evitar distracciones, su atención al material específico y los esfuerzos que realiza a lo largo de todo el proceso (Cartagena, citado por Mondragón, Cardoso y Bobadilla, 2017).

Respecto al rendimiento académico, Kaczynska (1986) afirma que es el fin de todos los esfuerzos $\mathrm{y}$ todas las iniciativas educativas que plantea el docente, de los padres de familia y de los estudiantes; la valoración de la escuela y el docente son juzgados por los aprendizajes y conocimientos que los estudiantes hayan adquirido.

Del mismo modo, Chadwick (1979) define al rendimiento académico como el desarrollo de capacidades y de características psicológicas que logra el estudiantes y se obtienen mediante el proceso de enseñanza - aprendizaje que le permite obtener un nivel de funcionamiento $y$ logros académicos en un determinado periodo, que se sintetiza en un calificativo final (cuantitativo en la mayoría de los casos) el cual describe el nivel alcanzado.

Finalmente Touron (1984) dice que el rendimiento es la calificación cuantitativa y cualitativa, que si es consistente y válida reflejará el logro de un determinado aprendizaje o de unos objetivos preestablecidos, es fundamental nos posibilita ver en qué medida los estudiantes han alcanzado cumplir con los objetivos educativos, no sólo sobre los aspectos de tipo cognoscitivos sino en muchos otros aspectos; puede permitir conseguir información para establecer estándares.
El objetivo de la presente investigación fue determinar la relación que existe entre los hábitos de estudio y el rendimiento académico de los estudiantes del nivel secundario de la Institución Educativa 52191 "Santo Domingo de Guzmán” del distrito de Laberinto, Tambopata.

\section{MÉTODOS Y MATERIALES}

La investigación estuvo enmarcada dentro del enfoque cuantitativo debido a que se recolectó datos y se realizó su análisis utilizando la estadística para probar hipótesis que se definieron con anterioridad (Sánchez, Reyes y Mejía, 2018); el diseño fue no experimental ya que las variables hábitos de estudio y rendimiento académico no fueron manipuladas, sino observadas como se presentan en su entorno y luego fueron analizadas (Hernández, Fernández y Baptista, 2014) y el tipo fue correlacional de corte transversal ya que se determinó la relación existente entre las variables de estudio a través de coeficientes (Bisquerra, 2009) para lo cual se recolectaron datos en un único momento.

La población de estudio fue integrada por el total de estudiantes (166) del nivel secundario matriculados en la Institución Educativa 52191 "Santo Domingo de Guzmán” del distrito de Laberinto en el año académico 2018. Con relación a la muestra, fue conformada por 116 estudiantes, cantidad obtenida mediante un muestreo probabilístico estratificado.

Para recoger información sobre la variable hábitos de estudio se utilizó la técnica de la encuesta y para la variable rendimiento académico, el análisis documental. Con relación a los instrumentos, para medir los hábitos de estudio se usó el Inventario de Hábitos de Estudios CASM-85. Revisión-98 elaborado por Vicuña (1985). Este instrumento consta de 53 ítems distribuidos en una escala dicotómica.

Está conformado por 5 dimensiones: Forma de estudio, resolución de tareas, preparación de exámenes, forma de escuchar la clase y acom- 
pañamiento al estudio. Finalmente, para recoger información sobre el rendimiento académico se utilizó el acta consolidada de evaluación integral del nivel secundario que recoge información so- bre las calificaciones obtenidas por los estudiantes en las áreas curriculares propuestos por el Diseño Curricular Nacional.

\section{RESULTADOS}

Luego de aplicar los instrumentos a los docentes se realizó la calificación de los mismos de acuerdo a sus pautas de corrección establecidas y se sistematizó para presentarlos de la siguiente manera:

\begin{tabular}{lcc} 
Tabla 1 \\
Resultados descriptivos de la variable hábitos de es \\
\hline \multicolumn{1}{c}{ Categorías } & Frecuencia & Porcentaje \\
\hline Inadecuado & 73 & 62,9 \\
Medianamente adecuado & 42 & 36,2 \\
Adecuado & 1 &, 9 \\
Total & 116 & 100,0
\end{tabular}

Fuente: Base de datos

En la tabla 1 se puede apreciar que el 62,9\% de estudiantes del nivel secundario presentan hábitos de estudio inadecuados, el 36,2\% presentan hábitos de estudio medianamente adecuados y solo el 0,9\% de los estudiantes tienen hábitos de estudio adecuados.

\section{Tabla 2}

Resultados descriptivos de la variable rendimiento académico

\begin{tabular}{lcc}
\hline \multicolumn{1}{c}{ Categorías } & Frecuencia & Porcentaje \\
\hline En inicio & 12 & 10,3 \\
En proceso & 41 & 35,3 \\
Logro previsto & 60 & 51,7 \\
Logro destacado & 3 & 2,6 \\
Total & 116 & 100,0 \\
\hline
\end{tabular}

Fuente: Base de datos

De acuerdo a la tabla 2, el 51,7\% de estudiantes del nivel secundario se encuentra en el nivel logro previsto, el 35,3\% se encuentra en el nivel en proceso, el 10,3\% está en el nivel en inicio y solo el 2,6\% se encuentra en el nivel logro destacado. 
Tabla 3

Matriz de correlación entre las variables hábitos de estudios y rendimiento académico

\begin{tabular}{llcc}
\hline & & $\begin{array}{c}\text { Hábitos de } \\
\text { estudio }\end{array}$ & $\begin{array}{c}\text { Rendimiento } \\
\text { académico }\end{array}$ \\
\hline \multirow{2}{*}{ Hábitos de estudio } & Correlación de Pearson & 1 &, $843^{* *}$ \\
& Sig. (bilateral) & 116 & 116 \\
& $\mathrm{~N}$ &, $843^{* *}$ & 1 \\
Rendimiento & Correlación de Pearson &, 000 & 116 \\
académico & Sig. (bilateral) & 116 & 160 \\
\hline
\end{tabular}

**. La correlación es significativa en el nivel 0,01 (2 colas).

Fuente: Base de datos

En la tabla 3 se puede ver que el coeficiente de correlación $r$ de Pearson entre las variables hábitos de estudio y rendimiento académico es de 0,843 con un p-valor inferior al nivel de significancia $(\mathrm{p}=0,000<0,05)$. Por lo expuesto, se concluye que existe una correlación alta, positiva y significativa entre las variables analizadas.

Tabla 4

Matriz de correlación entre las dimensiones de la variable hábitos de estudio y el rendimiento académico

\begin{tabular}{llc}
\hline & & $\begin{array}{c}\text { Rendimiento } \\
\text { académico }\end{array}$ \\
\hline \multirow{2}{*}{ Forma de estudio } & Correlación de Pearson &, $346^{\star \star}$ \\
& Sig. (bilateral) &, 000 \\
& $\mathrm{~N}$ & 116 \\
\hline \multirow{3}{*}{ Resolución de tareas } & Correlación de Pearson &, $609^{\star \star}$ \\
& Sig. (bilateral) &, 000 \\
& $\mathrm{~N}$ & 116 \\
\hline \multirow{3}{*}{ Preparación de exámenes } & Correlación de Pearson &, $637^{\text {** }}$ \\
& Sig. (bilateral) &, 000 \\
& $\mathrm{~N}$ & 116 \\
\hline \multirow{2}{*}{ Forma de escuchar la clase } & Correlación de Pearson &, $660^{\star \star}$ \\
& Sig. (bilateral) &, 000 \\
& $\mathrm{~N}$ & 116 \\
\hline \multirow{2}{*}{ Acompañamiento al estudio } & Correlación de Pearson &, $560^{\star \star}$ \\
& Sig. (bilateral) &, 000 \\
& $\mathrm{~N}$ & 116 \\
\hline
\end{tabular}

${ }^{\star \star}$. La correlación es significativa en el nivel 0,01 (2 colas).

Fuente: Base de datos 
En la tabla 4 se puede apreciar que existe correlación positiva, moderada y significativa entre las dimensiones resolución de tareas $(r=0,609$; $\mathrm{p}=0,000<0,05)$; preparación de exámenes $(\mathrm{r}=0,637 ; \mathrm{p}=0,000<0,05)$; forma de escuchar la clase $(r=0,660 ; p=0,000<0,05)$ y acompañamiento al estudio $(r=0,560 ; p=0,000<0,05)$ y la variable rendimiento académico. Por otra parte, también se puede ver que existe relación positiva, baja y significativa entre la dimensión forma de estudio y la variable rendimiento académico. $(r=0,346 ; p=0,000<0,05)$. En ese sentido se puede afirmar que la dimensión que más se relaciona con el rendimiento académico es la forma de escuchar la clase.

\section{DISCUSIÓN}

El objetivo de la presente investigación fue determinar la relación que existe entre los hábitos de estudio y el rendimiento académico de los estudiantes del nivel secundario de la Institución Educativa 52191 "Santo Domingo de Guzmán" del distrito de Laberinto, Tambopata.

Un primer hallazgo indica que la mayoría de estudiantes del nivel secundario presentan hábitos de estudio inadecuados. Esto significa que los estudiantes del nivel secundario no organizan su tiempo, espacio, técnicas y métodos para estudiar, lo cual genera que no utilicen estrategias e improvisen al momento de realizar sus actividades escolares, lo cual no favorece a su rendimiento escolar. Estos resultados coinciden con la investigación de Méndez (2004) quien halló que los estudiantes no practican hábitos de estudio; de Ramírez (2015) quien llegó a la conclusión que los hábitos de estudios de la mayoría de estudiantes son inadecuados; y de Huayta y Huayllani (2014) quienes indican que los hábitos de estudio tienen una tendencia negativa.

Con relación al rendimiento académico, la mayor parte de estudiantes del nivel secundario se encuentra en el nivel logro previsto, sin embargo, existe una considerable cantidad que se encuentran en proceso y en inicio, por lo que resulta ne- cesario que los docentes acompañen y brinden el apoyo necesario para que mejoren su rendimiento.

En la tabla 3 se halló que existe una correlación alta, positiva y significativa entre las variables analizadas $(\mathrm{p}=0,843 ; \mathrm{p}=0,000<0,05)$. Ello indica que si los estudiantes organizan su tiempo, espacio, técnicas y métodos para estudiar, se desempeñarán de forma adecuada y su rendimiento académico será óptimo en comparación a los estudiantes que no practican hábitos de estudio.

Estos resultados coinciden con la investigación de Argentina (2013) quien encontró que los estudiantes que resultaron con la mayor presencia de hábitos han obtenido mayor rendimiento por lo que se puede decir que a mayor presencia de hábitos de estudio, se obtendrá un mejor rendimiento académico y a menos presencia menor será el mismo; de Cepeda (2012) quien determinó que y la puesta en práctica de hábitos de estudio influye en el rendimiento académico de forma positiva, ya que los estudiantes que poseen ciertos hábitos de estudio que benefician su formación educativa y con ello su rendimiento académico; de Cortés (2017) quien mediante las pruebas de hipótesis de independencia, Chi cuadrado (X2) y la Correlación de Pearson ( $\mathrm{r}$ ), determinó que existe un grado de relación moderado de 0.475 , entre los hábitos de estudio y el rendimiento académico de los estudiantes, confirmando, al igual que otras investigaciones, la existencia de una relación de influencia entre las variables; de Ramírez (2015) quien halló que existe relación significativa entre hábitos de estudio y rendimiento académico de los estudiantes de la Escuela Superior de Formación Artística Pública Lorenzo Luján Darjón del distrito de Iquitos, provincia de Maynas, departamento de Loreto, durante el año 2011; de Villanueva (2015) quien concluyó que existe correlación positiva considerable entre las variables de estudio, donde el coeficiente de correlación de Pearson es 0,720; y de Jara (2015) quien halló que existe relación significativa entre los hábitos de estudio y el rendimiento académico en estudiantes del $2^{\circ}$ grado de Educación Secundaria de la 
Institución Educativa "Jesús Obrero" distrito de Comas.

Finalmente, se halló que existe correlación positiva, moderada y significativa $(p=0,000<0,05)$ entre las dimensiones de los hábitos de estudio y el rendimiento académico. Este hallazgo es corroborado por la investigación de Ortega (2012) así como de Villanueva (2015) quienes hallaron que las dimensiones de la variable hábitos de estudio se correlacionan significativamente con la variable rendimiento académico.

\section{CONCLUSIONES}

Se ha identificado que el nivel predominante de los hábitos de estudio de los estudiantes del nivel secundario de la Institución Educativa 52191 "Santo Domingo de Guzmán" del distrito de Laberinto, Tambopata es el nivel inadecuado, representado por el $62,9 \%$.

Se ha identificado que el nivel predominante del rendimiento académico de los estudiantes del nivel secundario de la Institución Educativa 52191 "Santo Domingo de Guzmán" del distrito de Laberinto, Tambopata es el nivel logro previsto representado por el $51,7 \%$.

Se ha determinado que existe una correlación alta, positiva y significativa entre las variables hábitos de estudio y rendimiento académico. El coeficiente de correlación $r$ de Pearson es de 0,843 con un p-valor inferior al nivel de significancia $(\mathrm{p}=0,000<0,05)$.

Se ha determinado que existe relación directa y significativa entre las dimensiones formas de estudio, resolución de tareas, preparación de exámenes, forma de escuchar la clase y acompañamiento al estudio con la variable rendimiento académico. Los coeficientes de correlación $\mathrm{r}$ de Pearson son de 0,$346 ; 0,609 ; 0,637 ; 0,660$ y 0,560 respectivamente.

\section{REFERENCIAS BIBLIOGRÁFICAS}

Argentina, A. (2013). Relación entre hábitos de estudio y rendimiento académico en estudiantes de primero básico. (Tesis de pregrado). Universidad Rafael Landívar, Quetzaltenango, Guatemala.

Belaúnde, I. (1994). Hábitos de estudio. Revista de la Facultad de Psicología de la Universidad Femenina del Sagrado Corazón, 2(2), pp. $14-21$.

Bisquerra, R. (2009). Metodología de la Investigación Educativa. (2a ed.). Madrid: La Muralla S.A.

Cepeda, I. (2012). Influencia de los hábitos de estudio en el rendimiento académico de los estudiantes. (Tesis de Maestría). Universidad de Guayaquil, Guayaquil, Ecuador.

Chadwick, C. (1979). Teorías del aprendizaje. Santiago: Ed. Tecla.

Chilca, M. (2017). Autoestima, hábitos de estudio y rendimiento académico en estudiantes universitarios. Propósitos y Representaciones, 5(1), pp. 71 - 99. DOI: http:// dx.doi.org/10.20511/pyr2017.v5n1.145

Cortés, J. (2017). Influencia de los hábitos de estudio en el rendimiento académico en estudiantes de $\mathrm{V}$ semestre de Contaduría Pública de la UPTC seccional Chiquinquirá periodos académicos 2015 - 2016. (Tesis de Maestría). Universidad Pedagógica y Tecnológica de Colombia, Chiquinquirá, Colombia.

Cruz, F. (2011). Hábitos de estudio, actitudes y autoestima relacionados con rendimiento académico en estudiantes de Enfermería. Cuadernos de Educación y Desarrollo, 3 (23). Recuperado de http://www.eumed.net/rev/ced/23/fcn.htm

Escurra, C., Romero, R., Moreno, B., Ahumada, C., Juárez, J. y Ramos, E. (2015). Hábitos de Estudio y Rendimiento Académico en los Estudiantes de la E.A.P. Estadística e Informática - UNJFSC - 2015. Big Bang Faustiniano, 5(2), pp. 11 - 17. Recuperado de http://revistas.unjfsc.edu.pe/ index.php/BIGBANG/article/view/13/13

Espinoza, J. (2017). Relación entre los hábitos de estudio y el rendimiento académico de los alumnos de escuelas profesionales acreditadas. Tzhoecoen, 9(4), pp. 29 - 40. Recuperado de http://revistas.uss.edu.pe/index.php/tzh/article/ view/721

Grados, J. y Alfaro, R. (2013). Hábitos de estudio y rendimiento académico en estudiantes del $1^{\circ}$ año de Psicología de la Universidad Peruana Unión, Lima, Perú. Revista Científica de Ciencias de la Salud, 6(2), pp. 48 - 53. Recuperado de https://revistas.upeu.edu.pe/index.php/RCCS/ article/view/128/137 
Hernández, R., Fernández, C. y Baptista, P. (2010). Metodología de la investigación. México: Mc Graw Hill.

Hernández, C., Rodríguez, N. y Vargas, A. (2012). Los hábitos de estudio y motivación para el aprendizaje de los alumnos en tres carreras de ingeniería. Revista de Educación Superior, 41(3), pp. 67 - 87. Recuperado de https:// www.redalyc.org/pdf/604/60425380005.pdf

Huayta, R. y Huayllani, Y. (2014). Influencia de los hábitos de estudio en el rendimiento escolar de los estudiantes de primero a quinto grado de educación secundaria en el área de Historia, Geografía y Economía de la institución educativa privada Santiago Ramón y Cajal del distrito de Uchumayo. (Tesis de Maestría). Universidad Nacional de San Agustín, Arequipa, Perú.

Jara, G. (2015). Hábitos de estudio y rendimiento académico en estudiantes de segundo grado de educación secundaria de la institución educativa "Jesús Obrero" distrito de Comas - 2015. (Tesis de Maestría). Universidad César Vallejo, Lima, Perú.

Kaczynska, M. (1986). El rendimiento académico y la inteligencia. Buenos Aires: Paidós.

Méndez, S. (2004). Relación entre los hábitos de estudio y el rendimiento académico en los alumnos de la Preparatoria núm. 22 de la Universidad Autónoma de Nuevo León. (Tesis de Maestría). Universidad Autónoma de Nuevo León, Nuevo León, México.

Mondragón, C., Cardos, D. y Bobadilla S. (2017). Hábitos de estudio y rendimiento académico. Caso estudiantes de la licenciatura en Administración de la Unidad Académica Profesional Tejupilco, 2016. RIDE. Revista Iberoamericana para la Investigación y el Desarrollo Educativo, 8(15), 661 -685 .

Ortega, V. (2012). Hábitos de estudio y rendimiento académico en estudiantes de segundo de secundaria de una institución educativa del Callao. (Tesis de Maestría). Universidad San Ignacio de Loyola, Lima, Perú.

Pineda, O. y Alcántara, N. (2017). Hábitos de estudio y rendimiento académico en estudiantes universitarios. Innovare, 6(2), pp. $19-34$.

Ramírez, H. (2015). Los hábitos de estudio en el rendimiento académico de los estudiantes de la Escuela Superior de Formación Artística Pública Lorenzo Luján Darjón de Iquitos, durante el año 2011. (Tesis doctoral). Universidad Nacional Mayor de San Marcos, Lima, Perú

Sánchez, H., Reyes, C. y Mejía, K. (2018). Manual de términos en investigación científica, tecnológica y humanística. Universidad Ricardo Palma: Lima. Recuperado de http:// repositorio.urp.edu.pe/handle/URP/1480
Touron, F. (1984). Factores del rendimiento académico en la Universidad. España: EUNSA.

Vicuña, L. (1998). Inventario de hábitos de estudio. Lima: CEDEIS.

Villanueva, R. (2015). Hábitos de estudio y rendimiento académico en el área de matemática de los estudiantes del V ciclo, de la Institución Educativa "Santa Rosa" No 80444, distrito de Santiago de Challaspataz en el año 2013. (Tesis de Maestría). Universidad Nacional de Educación "Enrique Guzmán y Valle”, Lima, Perú.

Yamada, G., Bazán, V. y Espinoza, J. (2017). Relación entre hábitos de estudio y motivación para el aprendizaje con el rendimiento académico en estudiantes de medicina veterinaria. Big Bang Faustiniano, 7(2), pp. 1-10. Recuperado de http://revistas.unjfsc.edu.pe/index.php/INFINITUM/ article/view/423/399 